\title{
Erratum to: Methane Emissions from Created Riverine Wetlands
}

\author{
Amanda M. Nahlik • William J. Mitsch
}

Published online: 8 March 2011

(C) Society of Wetland Scientists 2011

\section{Erratum to: Wetlands \\ DOI 10.1007/s13157-010-0038-6}

Note: An internal programming error resulted in higher values of methane emissions reported in this paper by a consistent factor of 1.4. The general findings and conclusions are not affected by this error.

\footnotetext{
Abstract

P.783, L.7-8: "19 and $68 \mathrm{~g} \mathrm{CH}_{4}-\mathrm{C} \mathrm{m}^{-2} \mathrm{y}^{-1}$ " should read " 13 and $47 \mathrm{~g} \mathrm{CH}_{4}-\mathrm{C} \mathrm{m}^{-2} \mathrm{y}^{-1}$ ",

P.783, L.8-9: "6 and $17 \mathrm{~g} \mathrm{CH}_{4}-\mathrm{C} \mathrm{m}^{-2} \mathrm{y}^{-1}$ " should read "4 and $12 \mathrm{~g} \mathrm{CH}_{4}-\mathrm{C} \mathrm{m}^{-2} \mathrm{y}^{-1}$ "

P.783, L.17: "82 $\mathrm{g} \mathrm{CH}_{4}-\mathrm{C} \mathrm{m}^{-2} \mathrm{y}^{-1}$ " should read " $57 \mathrm{~g}$ $\mathrm{CH}_{4}-\mathrm{C} \mathrm{m}^{-2} \mathrm{y}^{-1,}$

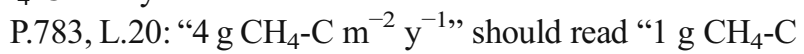
$\mathrm{m}^{-2} \mathrm{y}^{-1,}$

P.783, L.20: " $16 \mathrm{~g} \mathrm{CH}_{4}-\mathrm{C} \mathrm{m}^{-2} \mathrm{y}^{-1}$ " should read "7 $\mathrm{g} \mathrm{CH}_{4}-\mathrm{C}$ $\mathrm{m}^{-2} \mathrm{y}^{-1,}$
}

The online version of the original article can be found at http:// dx.doi.org/10.1007/s13157-010-0038-6.

\section{A. M. Nahlik $(\triangle) \cdot$ W. J. Mitsch}

Wilma H. Schiermeier Olentangy River Wetland Research Park,

Environmental Science Graduate Program and School

of Environment and Natural Resources,

The Ohio State University,

352 W. Dodridge Street,

Columbus, OH 43202, USA

e-mail: nahlik.amanda@epa.gov

W. J. Mitsch

e-mail: mitsch.1@osu.edu

Present Address:

A. M. Nahlik

U.S Environmental Protection Agency,

National Health and Environmental Effects Research Laboratory,

Western Ecology Division,

200 SW 35th Street,

Corvallis, OR 97333, USA

\section{Results}

P.786, L.80: "19 (68) and 6 (17) $\mathrm{g} \mathrm{CH}_{4}-\mathrm{C} \mathrm{m}^{-2} \mathrm{y}^{-1}$ ", should read "13 (47) and 4 (12) $\mathrm{g} \mathrm{CH}_{4}-\mathrm{C} \mathrm{m}^{-2} \mathrm{y}^{-1}$ ",

P.787, L.1: "80 (151) $\mathrm{mg} \mathrm{CH}_{4}-\mathrm{C} \mathrm{m}^{-2} \mathrm{~d}^{-1}$ " should read “55 (105) $\mathrm{mg} \mathrm{CH}_{4}-\mathrm{C} \mathrm{m}^{-2} \mathrm{~d}^{-1}$ "

P.787, L.1-2: "more than 4 times higher" should read "more than 2 times higher"

P.787, L.3: "more than 8 times higher" should read "more than 6 times higher"

P.787, L.7: "135 (277) and 87 (297) $\mathrm{mg} \mathrm{CH}_{4}-\mathrm{C} \mathrm{m}^{-2} \mathrm{~d}^{-1}$ " should read "94 (192) and 60 (206) $\mathrm{mg} \mathrm{CH}_{4}-\mathrm{C} \mathrm{m}^{-2} \mathrm{~d}^{-1}$ ",

P.787, L.12-13: "37 (122) $\mathrm{mg} \mathrm{CH}_{4}-\mathrm{C} \mathrm{m}^{-2} \mathrm{~d}^{-1}$ " should read " 25 (85) $\mathrm{mg} \mathrm{CH}_{4}-\mathrm{C} \mathrm{m}^{-2} \mathrm{~d}^{-1}$ ",

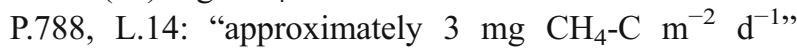
should read "approximately $2 \mathrm{mg} \mathrm{CH} \mathrm{CH}_{4}-\mathrm{C} \mathrm{m}^{-2} \mathrm{~d}^{-1}$ ",

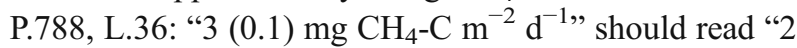
(0.1) $\mathrm{mg} \mathrm{CH}_{4}-\mathrm{C} \mathrm{m}^{-2} \mathrm{~d}^{-1}$,

P.788, L.39-40: "37 (36) $\mathrm{mg} \mathrm{CH} \mathrm{CH}_{4}-\mathrm{C} \mathrm{m}^{-2} \mathrm{~d}^{-1}$ and 77 (160) $\mathrm{mg} \mathrm{CH}_{4}-\mathrm{C} \mathrm{m}^{-2} \mathrm{~d}^{-1}$ " should read "26 (25) $\mathrm{mg} \mathrm{CH}_{4}-\mathrm{C}$ $\mathrm{m}^{-2} \mathrm{~d}^{-1}$ and 53 (111) $\mathrm{mg} \mathrm{CH}_{4}-\mathrm{C} \mathrm{m}^{-2} \mathrm{~d}^{-1}$ "

P.788, L.49-50: "23 (82) $\mathrm{g} \mathrm{CH}_{4}-\mathrm{C} \mathrm{m}^{-2} \mathrm{y}^{-1}$-the mean rate of which is over 2.5 times" should read "16 (57) $\mathrm{g}$ $\mathrm{CH}_{4}-\mathrm{C} \mathrm{m}^{-2} \mathrm{y}^{-1}$-the mean rate of which is over $15 \%$ "

\section{Table 1}

Methane Emission $\left(\mathrm{g} \mathrm{CH}_{4}-\mathrm{C} \mathrm{m}^{-2} \mathrm{y}^{-1}\right)$ should read 12, 47, and 29 for the Current Study 2006-2008

Wetland 1, Wetland 2, and ORW Mean, respectively. The Current Study 2006-2008 Natural Wetland methane Emission $\left(\mathrm{g} \mathrm{CH}_{4}-\mathrm{C} \mathrm{m}^{-2} \mathrm{y}^{-1}\right)$ should read 57.

\footnotetext{
Discussion

P.789, L.50: "42 $\mathrm{g} \mathrm{CH}_{4}-\mathrm{C} \mathrm{m}^{-2} \mathrm{y}^{-1}$ " should read " $29 \mathrm{~g}$ $\mathrm{CH}_{4}-\mathrm{C} \mathrm{m}^{-2} \mathrm{y}^{-1}$ ”

P.790, L.33: "16 $\mathrm{g} \mathrm{CH}_{4}-\mathrm{C} \mathrm{m}^{-2} \mathrm{y}^{-1}$ " should read "7 $\mathrm{g}$ $\mathrm{CH}_{4}-\mathrm{C} \mathrm{m}^{-2} \mathrm{y}^{-1}$
} 
P.790, L.35: "4 $\mathrm{g} \mathrm{CH}_{4}-\mathrm{C} \mathrm{m}^{-2} \mathrm{y}^{-1}$ " should read " $1 \mathrm{~g} \mathrm{CH}_{4}-\mathrm{C}$ $\mathrm{m}^{-2} \mathrm{y}^{-1}$

P.791, L.8: "28 to $49 \mathrm{~g} \mathrm{CH}_{4}-\mathrm{C} \mathrm{m}^{-2} \mathrm{y}^{-1}$ " should read "28 to $34 \mathrm{~g} \mathrm{CH}_{4}-\mathrm{C} \mathrm{m}^{-2} \mathrm{y}^{-1,}$

P.791, L. 14: "3 $\mathrm{g} \mathrm{CH}_{4}-\mathrm{C} \mathrm{m}^{-2} \mathrm{y}^{-1}$ " should read " $2 \mathrm{~g} \mathrm{CH}_{4}-\mathrm{C}$ $\mathrm{m}^{-2} \mathrm{y}^{-1,}$
P.791, L.71: "82 $\mathrm{g} \mathrm{CH}_{4}-\mathrm{C} \mathrm{m}^{-2} \mathrm{y}^{-1 "}$ should read "57 $\mathrm{g}$ $\mathrm{CH}_{4}-\mathrm{C} \mathrm{m}^{-2} \mathrm{y}^{-1,}$

P.792, L.2: "in 25 years, or 40 years" should read "in 55 years, or 70 years"

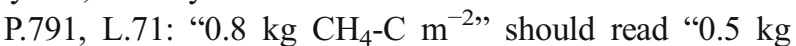
$\mathrm{CH}_{4}-\mathrm{C} \mathrm{m}^{-2}$, 